\title{
REVIEW
}

\section{SELF-PROPELLED AFFINITY BIOSENSORS: MOVING THE RECEPTOR AROUND THE SAMPLE}

\author{
J. Wang* \\ Department of Nanoengineering, University of California San Diego, San Diego, CA 92903, \\ USA
}

\footnotetext{
* To whom correspondence is to be addressed

*e-mail: josephwang@ucsd.edu
}

\begin{abstract}
Self-propelled nanomotors offer considerable promise for developing novel biosensing protocols involving 'on-the-fly' recognition events. This article reviews recent advances in using catalytic nanomotors for bioaffinity sensing and for isolating target biomolecules and cells from complex biological samples. A variety of receptors, attached to self-propelled nanoscale motors, can thus move around the sample and, along with the generated microbubbles, lead to greatly enhanced fluid transport and accelerated recognition process. Such operation addresses the challenges imposed by the slow analyte transport in designing sensitive bioaffinity assays. The recognition element can be attached onto the motor surface or embedded in the motor material itself. Receptor-functionalized nanomotors based on different biomolecular interactions have thus been shown extremely useful for rapid target isolation from complex biological samples without preparatory and washing steps. Tubular microengine microtransporters, functionalized with antibody, ss-DNA, aptamer or lectin receptors, are particularly useful for direct detection and isolation of proteins, nucleic acids, proteins or cancer cells. Micromotors with 'built-in' recognition, exploiting the selective binding properties of the outer layer of such micronegines, can also be used. Greatly enhanced analyte-receptor interactions can also be achieved through
\end{abstract}


the increased fluid transport associated with the movement of unmodified micromotors. The attractive features of the new motion-based bioaffinity sensing and separation protocols open up new opportunities for diverse biomedical, environmental and security applications.

Keywords: Nanotechnology, Bioaffinity, Nanomachines, Biosensors, Nanomotors

\section{Introduction}

Locomotion of nanoscale objects through fluid environments is one of the most exciting fields of nanotechnology (Wang, 2013; Guix et al, 2014, Paxton et al, 2004; Ozin 2005; Mei et al, 2008; Pumera 2010 (Reviewer 2)). Recent efforts have led to the development of a wide range of nanomotors based on different propulsion mechanisms. These include chemically-powered motors that convert chemical energy into autonomous motion (Paxton et al, 2004; Mei et al, 2011; Sanchez et al, 2015; Solovev et al, 2009) and fuel-free motors that rely on variety of external stimuli, such as magnetic or ultrasound actuations (Peyer et al, 2013; Kagan et al, 2012). Such synthetic nano/microscale motors represent a major step towards the development of practical nanomachines. Advanced fabrication schemes used for creating such nanomachines can impart new functionalities and capabilities. These recent advances and new capabilities have opened up new sensing opportunities and bioanalytical applications. These involve not only new sensing applications in previously inaccessible microscale environments but primarily fundamentally new sensing approaches based on the motor movement.

This article reviews recent advances in using self-propelled nanomotors for bioaffinity sensing and for isolating target biomolecules and cells from complex body fluids. Bioffinity sensors, that exploit selective recognition of specific target species for triggering useful signals, represent a major class of modern biosensors (Cosnier, 2005; Wang, 2006; Merkoci 2013). While low detection limits are required to facilitate early disease detection, such ultrasensitive detection remains a major challenge to bioaffinity assays in view of the ultrasmall sample volumes. Common bioaffinity sensors rely on placing the sample droplet over the receptormodified transducer and measuring the extent of the analyte-receptor interaction following an incubation period under quiescent conditions. Such droplet-based bioassays thus rely solely on diffusion transport. Fundamental studies revealed that the detection limits for such bioaffinity assays reflect the analyte transport limitations, and not a signal transduction limitation (Sheehan and Whitman, 2005). Enhancing the mass transport by using convection, e.g., solution stirring, is 
often challenging for increasing the response of such microassays. Attempts in this direction have included acoustic streaming, air-driven bladders, or moving boundaries (Links, 2012; Adey et al, 2002; Morales-Narváez 2014). (Reviewer 1, Comment 1). Bioaffinity interactions are also widely used for separating and isolating biological targets from raw biological samples. Such isolation processes are extremely challenging owing to the complexity of body fluids and rely on laborious time-consuming procedures.

The motion-based biosensing strategy relies on the continuous movement receptor-modified microengines through such complex samples in connection to diverse 'on-the-fly' biomolecular interactions. Such movement of the receptor around a complex sample promotes its interaction with the target analytes and represents a fundamentally new paradigm in analytical chemistry. In particular, bubble-propelled catalytic micromotors, functionalized with different bioreceptors, described in this article, provide a new 'on-the-fly' approach for bioaffinity assays and for isolating target biomolecules from complex biological samples and transporting them to a clean environment for downstream analysis (Campuzano et al, 2011; Kagan et al, 2011; RestrepoPérez et al, 2014). Movement of the bioreceptor around the sample leads to a new approach for bioaffinity assays and bioseparations that addresses the limitations associated with the slow analyte transport under quiescent conditions used in such microassays (Figure 1A). Unmodified self-propelled micromotors can also enhance greatly analyte-receptor interactions through the increased fluid transport and mixing associated with the motor movement and generated microbubbles tail (Orozco et al, 2014) (Figure 1B). Such mixing induced by the micromotor motion can assist the transport of the target molecule within the sample solution toward an immobilized receptor (Morales-Narváez 2014). Such motion-based sensing strategy thus holds considerable promise for diverse biosensing and biochip applications, involving a variety of selfpropelled receptor-modified microtransporters and involving different biomolecular interactions. The motor-based 'capture-and-transport' approach leads also to effective isolation of biological targets of different scales from unprocessed biological samples. 
The successful realization of the new motion-based bioaffinity sensing and separation concepts requires an optimal propulsion behavior and a large towing force of individual microtransporters in complex biological matrices along with control of their surface chemistry and directionality. We will thus discuss first the fabrication and operation of tubular microengines that are most commonly used in such motion-based biosensing and separation, followed by other practical considerations, novel sensing strategies, analytical performance and future prospects of these self-propelled biosensors.

\section{Tubular Microengine Transporters}

Among chemically-powered nanomotors, tubular microengines are particularly attractive for biosensing applications due to their efficient bubble-induced propulsion in relevant biological fluids containing hydrogen peroxide fuel (Mei et al, 2011, Sanchez et al., 2015). Bubblepropelled tubular microengines ('microrockets') have been developed to address the limitation of catalytic nanowire motors to low ionic-strength media. Such tubular microengines display high speed and power, along with a precise motion control and can be easily modified to impart new functionalities and capabilities. Tubular microengines have been commonly fabricated using standard photolithography or template electrosynthesis procedures (Mei et al, 2008; Gao et al, 2011). The resulting tubular microengines have an inner (catalytic) Pt surface, a diameter opening of 2-10 $\mu \mathrm{m}$ and are typically 5-100 $\mu \mathrm{m}$ long. The oxygen-bubble propulsion thrust of these tubular microengines is associated with the decomposition of the hydrogen peroxide fuel on the inner catalytic platinum surface. The conical shape of the microengine assists in the unidirectional bubble evolution, with small amounts of surfactant (e.g., 2\% sodium cholate) (Reviewer 1, Comment 3). are used to promote this bubble evolution and release. The movement of the micromotor can be controlled using external mechanisms, including magnetic or ultrasound fields, as well as temperature or light stimuli (Balasubramanian et al, 2009; Wang and Manesh 2010; Restrepo-Pérez et al, 2014; Rao et al, 2015). (Reviewer 1, Comment 2). For example, the incorporation of a magnetic layer (e.g., $\mathrm{Ni}$ ) during the preparation process allows for a precise magnetic navigation of their movement within narrow microchip channels. Tubular microengines based on metals others than platinum have been developed recently. In particular, zinc-based microengines have been developed for efficient propulsion in acidic (stomach) media 
(Gao et al, 2011) and were recently used for the first operation of micromotors in living organisms (Gao et al, 2015).

An outer gold or polymeric layer can be used to functionalize the tubular microengines with different bioreceptors (using the surface chemistry described below). Such surface functionalization reduces the speed of these micromotors from 500-1000 body-lengths per second $(\mathrm{bl} / \mathrm{s})$ for the bare microengines to $\sim 100 \mathrm{bl} / \mathrm{s}$ for receptor-functionalized motors in the presence of $1-5 \%$ of the peroxide fuel. (Reviewer 1; Comment 3) These modified motors display lower speeds, down to $20 \mathrm{bl} / \mathrm{s}$, in biological media.

Such movement of the bioreceptor around the sample leads to a fundamentally new approach for bioaffinity assays and bioseparations. In particular, the localized fluid convection and vortex streams, associated with the rapid movement of these self-propelled microtransporters and their bubble tail, lead to a favorable hydrodynamic environment which enhances the kinetics of the binding reaction and promotes the interaction of the target analytes with the receptor (Kagan et al, 2011; Orozco et al, 2014)(Figure 1). This 'built-in' induced fluid transport represents a unique advantage of the micromotor isolation strategy.

We examined of the effect of bubble-propelled tubular microengines on the enhanced diffusion of passive microsphere tracers (Orozco et al, 2014). The tubular microengines were found to substantially increase the displacement of such tracer microparticles through fluid convection induced by the bubbles produced during their propulsion. This study highlighted the key role played by the generated bubbles upon the increased fluid transport in the presence of tubular microengines. Because of the strong coherent flows driven by the rising bubble emerged from the motors, the enhanced transport of passive tracer and fluid motion observed in the presence of tubular microengines was found to be substantially larger than for other types of common catalytic micromotors, including as bimetallic nanowires and Pt-Janus microspheres. The increased fluid transport associated with such movement of functionalized motors and with generated bubbles greatly enhances the analyte-receptor interactions.

\section{Isolation of biomaterials based on receptor-functionalized microengine microtransporters}


The selective isolation of biological targets from untreated complex samples is a very challenging task that offers considerable promise for disease diagnosis or food safety. The 'capture-and-transport' operation of receptor-functionalized micromotors can offer the selective sorting of biological targets from complex samples (Campuzano et al, 2011; Restrepo-Pérez et al, 2014). Different biomolecular interactions have been used for separating biological targets of varying scales, ranging from the molecular to cellular levels. Target analytes can thus be captured and transported to a clean environment, thus avoiding laborious sample preparation steps. Such operation of receptor-functionalized micromotors can also replace the wash steps of common bioaffinity assays.

The sophisticated transport capabilities of protein motors (e.g., kinesin) have inspired the use of synthetic nanomotors for performing target isolation applications. Such biomotors are involved in intracellular transport of different cargos (e.g., vesicles) along microtubule filaments using ATP hydrolysis as energy source (Vale 2003). Motor proteins were used also for manipulating nanoscale components in artificial environments (van den Heuvel and Dekker, 2007; Schmidt and Vogel, 2010). For example, antibody and DNA-functionalized kinesin motors were used for transporting liposomes or nucleic acids along microtubule 'tracks' in engineered microsystems (Schmidt and Vogel, 2010; Hiyama et al, 2008, Fischer et al, 2009). However, the limited operational stability of protein motors in artificial environments and the need for microlithographic tracks to guide their movement, hinder their practical applications in complex biological samples.

The high propulsion power, ease of navigation and surface modification of catalytic microtransporters lay the foundation for the motor-based biosensing and isolation strategy. Bubble-propelled tubular microengines are particularly attractive for performing the challenging tasks of capture, transport and release specified cargoes. Functionalized microengines selectively capture the target in a complex sample and can be guided to a clean zone for subsequent detection of the captured target. In this way, such microengines are not only acting as selective microtransporters for the rapid target isolation from raw biological samples, but also creating the possibility of further quantification of the target analyte. Larger targets, e.g., cancer cells, can be captured and directly identified with an optical microscope (Balasubramanian et al, 2011). Different detection techniques can be used to monitor the binding event of small molecules (e.g., 
using fluorescent tracers). For example, a direct optical visualization of the captured target on moving microengines can be realized using a biotin-labeled detection molecule that can actively bind to streptavidin-coated fluorescent nanoparticle tags. The micromotor separation concept is applicable for the isolation of different types target biomolecules in connection to diverse surface receptors and biomolecular interactions (Figure 2). Note the direct visualization of binding and transport using the fluorescent tracers or the target cell itself.

It is important to note that the fuel and surfactant $\left(\mathrm{H}_{2} \mathrm{O}_{2}\right.$ and $\mathrm{NaCh}$, respectively), essential for the movement of microengines in biological fluids, do not noticeably affect the surface chemistry (required for receptor functionalization) and the corresponding biological interactions (binding event) at the levels involved. (Reviewer 1; Comment 3) Apart from this unique bubbling effect, the fast movement of the functionalized microtransporters across the samples increases the likelihood of target contact with the surface-confined receptor. It is apparent that the effective movement and power of tubular microengines along with their modification versatility, guided movement, and large towing force are extremely attractive for isolating biological materials from raw samples. Such target isolation and separation capability of receptor-modified tubular microengines offer attractive possibilities in different fields.

\section{Microengines with a 'Built-In' Recognition}

In addition to a separate receptor functionalization step, it is possible to exploit the 'built-in' recognition properties of the outer polymeric layer of the microengines. The selective binding properties of the outer layer of such bilayer tubular micromotors can thus be used for designing microtransporters with a 'built-in' recognition. Two such microengines routes, with a 'built-inrecognition', have been reported. For example, Wang and colleagues described a poly(3aminophenylboronic acid) (PAPBA)/Ni/Pt microtube engine for 'on-the-fly' separations of carbohydrates (Kuralay et al, 2012). These microengines combine the recognition of monosaccharides by the boronic-acid based outer polymeric layer with the catalytic function of the inner Pt layer for the capture and transport of target sugars. Such recognition relies on the well-established complexation of monosaccharides with the boronic acid ligand. Controlled release of captured yeast cells toward subsequent reuse was accomplished by the competitive binding of added fructose. 
Orozco et al demonstrated that molecular imprinting (MIP) represents an attractive route for creating of specific recognition sites in synthetic self-propelled nanomachines. (Orozco et al 2013). Tailor-made recognition cavities have thus been introduced into tubular engine microtransporters through cavities in the outer polymer layer by growing this layer in the presence of the analyte (template). This was accomplished by adsorbing first the positively charged protein template onto the walls of the membrane micropores, followed by electropymerization of the PEDOT/PSS outer layer. The resulting MIP microengines offered attractive capabilities for autonomous binding, directional transport and accumulation of the target protein template, including 'on-the-fly' separation from untreated saliva and serum samples. The template-imprinted microtransporter 'capture-transport' concept was illustrated using av-FITC as a model protein (Figure 3). Another molecularly imprinted microtransporters, based on Janus microsphere motors with specific molecular recognition ability, was also developed (Huang and Shen, 2014). Imprinted sites for the drug propranolol were introduced into Janus micromotors by cross-linking polymerization. The resulting self-propelled microtransporters could also be used as autonomous carriers for controlled drug delivery. The MIP-based micromotor concept it could be readily extended for the separation and isolation of diverse target analytes, offering great promise for biomedical and environmental applications.

\section{Receptor functionalization and related surface chemistry}

As with conventional bioaffinity sensors, the quality of the receptor-containing surface layer, on the moving microengine, is of extreme importance for achieving high analytical performance. A judicious modification of the surface of tubular micronengines is essential to ensure high binding efficiency, specificity and minimization of non-specific adsorption. Control of the surface chemistry and of the receptor density on the microengine is thus crucial for the success of the motor-based biosensing and bioseparation. Our effort have demonstrated the rational functionalization of tubular microengines with different receptors (Figure 2) and that such functionalization does not hinder their propulsion efficiency.

Two routes involving covalent interactions have been widely used for functionalizing tubular microengines with the bioreceptor. These include self-assembled monolayer (SAM) chemistry in connection to our gold surfaces (Steel et al, 1998) or via EDC/NHS coupling to 
carboxy moieties on outer polymeric layers (Garcia et al, 2013). Streptavidin-functionalized micromotors could also be used in connection to biotylated receptors. SAM chemistry is commonly used to modify the gold surface with a receptor selected in accordance to the target analyte (cells, nucleic acids, proteins and other biomarkers), by using specific surface chemistry protocols. In particular, mixed alkanethiol monolayers of mercaptoundecanoic (MUA)/mercaptohexanol (MCH) offer a balance among minimal non-specific interactions, high loading, and preferred orientation (Levicky et al, 1998). For example, attachment of an antibody can be accomplished through carboxyl-terminated groups of MUA via a EDC/NHS chemistry (Fig. 5a, b)(Briand et al, 2006). The surface of the microtransporters can be also modified with a binary SAM composed of thiolated oligonucleotides and short MCH spacers when DNA and aptamers are used as receptors. The MCH spacer induces "standing up" of these nucleic acid receptors to ensure optimal interaction and minimize non-specific adsorption (Levicky et al, 1998). Some diminution in the microengine's speed may occur due to some sulfur poisoning of the inner catalytic platinum surface. Instead of gold outer layers, it is possible to use mixed polymeric layers (e.g., poly(3,4-ethylenedioxythiophene) PEDOT/carboxy-PEDOT) to attach an antibody via a similar EDC/NHS chemistry. The mixed outer polymeric layer of the microengines can be prepared by co-electropolymerization of PEDOT:PEDOT-COOH (from solution containing a mixture of the monomers) followed by plating of the Ni and Pt layers (from a Pt/Ni mixture electroplating solution). Figure 4 displays the surface modification of such tubular microengine along with the cross section of such multi-layer rocket.

\section{Antibody-Functionalized Microengines for Selective Biodetection}

An attractive microchip immunoassay protocols, based on the movement of antibodyfunctionalized synthetic tubular microengines within microchannel networks, was developed by Wang's team (Garcia et al, 2013). As was described in the previous section, such polymer-Pt microengines contain carboxy moieties on their outer polymeric layer and can be functionalized through a common EDC/NHS chemistry. Polymer (PEDOT:PEDOT-COOH)/Ni/Pt immunomicroengines were thus used for an 'on-the-fly' microchip sandwich immunoassays involving antibody-functionalized microtransporters. Such immunoassays include directed capture and transport of target proteins along predetermined paths in the microchip system. The microchip sandwich immunoassay is based on self-propelled antibody-functionalized tubular 
micronengines able to perform 'on-the-fly' each of the immunoassay steps in different reservoirs. Tagging the antigen or a secondary antibody with a microsphere tag offers convenient direct optical visualization of the protein binding events. (e.g., Figure 5).

The autonomous transport of antibody-functionalized tubular microengines leads to 'onthe-fly' capture and isolation of the target proteins. All the capture-transport-tag-transport steps involved in the immunoassay protocol were thus carried out in the microfluidic device, hence replacing the common capture-wash-tag-wash sequence of traditional sandwich immunoassays (e.g, ELISA bioassays well plates). Such operation thus obviates the need for multiple wash steps and results in a simplified immunoassay protocol. Movement of the micrometer-size captured sphere tag or bacteria target thus offers convenient direct real-time optical visualization of the protein binding event. Such coupling of functionalized tubular micronegines, immunoassays, and microchip devices holds considerable promise for diverse applications of lab-on-a-chip systems.

The use of microsphere tracers of different sizes and shapes has opened up the possibility of developing powerful micromotor-based multiplexed immunoassays (Vilela et al, 2014). This multiplexed microchip immunoassay consists of micromotors functionalized with different antibodies for the selective recognition and capture of different microscopic gold wire-tagged target proteins. Tagging the target proteins with nanomaterials (wires, particles) of different shapes and sizes allowed direct visualization of the antigen-antibody recognition event. Such tagging the analyte with microparticle tracers of different sizes offers the unique opportunity for the direct visualization of the antibody-protein recognition event. While the micromotor-based multiplexed immunoassay was demonstrated for simulants of dangerous protein toxins, the concept can be readily extended to the development of effective systems that rapidly differentiate other types of coexisting biomolecules (e.g. nucleic acids).

Orozco et al described recently a micromotor-based approach capable of rapid screening, detecting, isolating and damaging biothreat agent simulant spores from nearly untreated samples (Orozco et al, 2015). The detection and elimination of the Bacilus anthracis, a rod-shaped, Gram-positive, saprophytic bacterium that cause anthrax, represent a major challenge to the biodefense community. The 'on-the-fly' motor-based spore detection protocol relies on the movement of anti-B. globigii antibody-functionalized tubular 
microengines in a contaminated solution to capture and transport single and multiple $B$. globigii spores (Figure 6). High selectivity against excess of non-target Staphylococcus aureus and Escherichia coli species was demonstrated, along with successful operation in common environments where spores can be found (e.g., lake or tap water). Subsequently, accelerated destruction of anthrax simulant spores was illustrated through greatly motorinduced enhanced mixing of mild quiescent oxidizing solutions.

Immunoassays can also greatly benefit from the convective transport induced by the motion of unmodified micromotors. Merkoçi and colleagues described a greatly enhanced microarraybased immunosensing based on mixing induced by the motion of self-propelled microengines (Morales-Narváez et al, 2014). Antibody microarrays were thus spotted as bioreceptors and the target was detected in samples containing self-propelled polymer/Pt tubular micronegines, which induce a mixing effect to improve mass transport. Such motor-based array concept can be extended to DNA and cell analyses.

\section{Capture and sorting of cancer cells at antibody-functionalized microtransporters}

Sanchez and colleagues described the possibility to use unmodified catalytic tubular Ti/Fe/Pt microengines to pick and direct neuronal $\underline{\mathrm{CAD}}$ animal cells in a controllable manner (Sanchez et al 2011a). The guided motion enabled the release of the loaded cell at a desired location. A more selective nanomotor-based cell sorting methodology can be accomplished using a receptormodified microengine. Such cell-sorting platform has been demonstrated by our team for the isolation of carcinoembryonic antigen $(\mathrm{CEA}+)$ expressing pancreatic cancer cells under physiological conditions (Balasubramanian et al, 2011). Since circulating cancer cells (CTC) are the primary entities responsible for spawning cancer metastasis, detecting and isolating trace levels of tumor cells in body fluids is extremely important for the diagnosis of cancer.

The new concept relies on the selective binding and transport ability of tubular microengines, functionalized with anti-CEA monoclonal antibody (mAb) (Figure 2, bottom). The bubble propulsion of the tubular microengines, combined with their high towing force, has enabled effective binding and transport of CTC in untreated serum. This was illustrated for the 'on-the-fly' capture and transport of a CEA+ pancreatic cancer cell by an anti-CEA mAb modified nanomotor in diluted human serum. Efficient movement of the cancer-cell loaded 
nanomotor over a pre-determined path was also demonstrated. The ability of these modified microengines to selectively recognize the target cancer cells in cell mixtures was also demonstrated The mAb-functionalized microtransporters have sufficient force $(>10 \mathrm{pN})$ to transport a relatively large cell even in diluted serum. The minimum estimated force necessary for transporting a $16 \mu \mathrm{m}$-diameter cancer cell at one body length per second is $2.5 \mathrm{pN}$.

\section{'On-the-Fly' DNA Detection at Functionalized Micoengines}

The detection of DNA hybridization can also benefit from the continuous movement of the oligonucleotide probe receptor through the sample. Such 'on-the-fly' detection of specific DNA sequences holds great promise for diagnosing infectious or genetic diseases. Our team has demonstrated that ss-DNA-functionalized microengines can act as selective transporters of complementary nucleic acids (synthetic DNA or bacterial rRNA), allowing for their rapid isolation directly from raw biological matrices such as serum, urine or saliva without preparatory and washing steps (Kagan et al, 2011). During their movement, the oligonucleotide probe, confined onto the moving micromotor surface, hybridizes with the corresponding nucleic acid target, allowing for its capture and transport. Tagging a secondary probe with a fluorescent tag offers convenient evaluation of the target loading and transport processes, through optical monitoring of the resulting hybrid. Pre-labelling of the target with a fluorescent tracer offer direct visualization of the 'on-the-fly' hybridization event (Figure 2, bottom). The fast movement and the corresponding efficient localized convection enhance dramatically the hybridization efficiency. Rapid (few minutes) saturation of the motor's surface with fluorescently-labeled nucleic acids can thus be observed. Sensitive detection of nanomolar levels of target DNA, without contributions from excess of mismatch or non-target sequences, have thus been demonstrated. The practical utility of the motor-based DNA isolation concept was illustrated also for the transport of E. coli $16 \mathrm{~S}$ rRNA from raw lysate sample solutions. The ability of the modified microengines to transport the captured DNA across a microchip channel, from a complex sample zone to a 'clean' zone, was also demonstrated. While no post-processing was carried out, one could imagine performing reverse transcriptase and polymerase chain reactions in the clean well to accurately quantify or sequence the target RNA.

A new 'signal-on' motor-based DNA sensing route was developed recently by Minteer's group (Nguyen and Minteer 2015). This elegant protocol relies on a tubular microengine that 
displays movement only in the presence of DNA target. Such "turn on" characteristic is achieved by the recruitment of $\mathrm{Pt}$ nanoparticle-DNA conjugate as motion-inducing catalyst for micromotors through DNA hybridization. Such hybridization-induced capture of the catalytic $\mathrm{Pt}$ nanoparticles leads to the motor movement in the presence of the peroxide fuel. Such 'SignalOn' micromotor approach could be extended to bioaffinity sensing of other biological targets. Simmchen et al. described another motion-based DNA detection involving direct visual tracking of cargo (Simmchen et al, 2012). The method relied on silica microparticles that were functionalized with catalase and single-stranded DNA. Without hybridization of the target DNA, the motor was not connected to the detection system (cargo). In contrast, the hybridization event led to a detectable movement of the cargo by optical microscopy. (Reviewer 1, Comment 4)

\section{Target Loading and Unloading of Using Microengines Functionalized with Aptamers or Lectins.}

Wang and coworkers demonstrated an aptamer-microtransporter platform for the selective binding and transport of target proteins (Orozco et al, 2011). The concept was illustrated by the highly selective isolation of thrombin from untreated biological samples (e.g., serum, plasma) using self-assembly of a mixed thiolated thrombin-ATP aptamer/MCH layer on the gold surface of tubular microengines. The results demonstrate the high selectivity towards the target thrombin protein with an effective discrimination against a large excess of non-target proteins. Quantitative isolation of protein targets was obtained using fluorescent tags and by adjusting the solution concentration and incubation time. Triggered release of the captured thrombin from the surface-bound mixed thrombin-ATP aptamer was accomplished in the presence of ATP.

A nanomotor bacteria isolation strategy involving the movement of ConA-functionalized gold/nickel/polyaniline/platinum tubular microengines to scour, interact and isolate pathogenic bacteria from distinct complex samples has been demonstrated (Campuzano et al, 2012). Controlled release of the captured bacteria from a moving microengine was achieved using a low-pH glycine solution able to dissociate the lectin-bacteria complex. Such ability to isolate and unload target bacteria is essential for identifying pathogenic bacteria serotypes. The practical utility of the new microengine approach was illustrated by the ability of the lectin-modified microengines to recognize the target bacteria in different $E$-coli-inoculated real samples, ranging 
from apple juice to seawater. The ability of the microengines to simultaneously capture and transport both the target bacteria and polymeric drug-carrier spheres toward "on-the-spot" therapeutic action was illustrated.

Towards nanomotor-based Lab-on-Chip devices Error! Bookmark not defined.

The integration of functional nanomotors into functional microchip devices could lead to a new generation of labs-on-a-chip systems with attractive analytical capabilities. The nanomotorbased target isolation protocol could be readily incorporated into microchannel networks for creating advanced microchip devices integrating autonomous capture-transport-release-detection operations. Such active transport of captured target molecules by self-propelled receptormodified microtransporters propelling in quiescent solutions within microchannel networks represent an attractive alternative to current pressure-driven or electrokinetic flow-based microfluidic devices. Protein motors were used previously in lab-on-a-chip formats. In particular, kinesin/microtubule-based molecular shuttles were used for the pick-up and guided transport of selected cargo (van den Heuvel and Dekker, 2007)

Synthetic nanomotors are highly compatible with engineered microsystems and can be readily adapted to different lab-on-a-chip platforms (Garcia et al, 2013; Restrepo-Pérez et al, 2014). The ability of different artificial nanomotors to transport variety of cargo through a microchannel network with or without a hydrostatic flow has been demonstrated (Burdick et al, 2008; Sanchez et al, 2011a). The precise motion control and large towing power allow the nanomotors to load multiple cargos and transport them through complex microchannel networks to desired locations in the microchip. Such advanced spatial motion control within the narrow channels and different reservoirs of the microchip reflects the precise magnetic guidance associated with its intermediate Ni layer. Movement of tubular microngines against flowing streams has been demonstrated (Sanchez et al, 2011a).

Future nanomotor-based lab-on-chip microsystems are expected to rely on functionalized nanomotors and integrating the capture-transport-tagging-cleanup-enrichment-detection-release (Reviewer 1) operations within spatially separated zones. Such autonomous microchip platform would contain multiple reservoirs (with sample and reagent/tracer inputs) through which the functionalized nanomotors can be directed for the rapid and simple isolation, manipulation, 
preconcentration and detection of multiple biological targets. The receptor-modified nanomotors would thus transfer the captured target from the sample reservoir, containing the complex biological fluid, into the detection reservoir with a pure buffer ideal for sensing. The captured biomarker would undergo various manipulation/processes in different zones on its way from the sample reservoir to the detection one. The entire microchip operation will be carried out in a quiescent solution without any bulk solution flow.

Sanchez and coworkers (Restrepo-Pérez et al, 2014) developed a microchip system in which self-propelled catalytic microengines are biofunctionalized and trapped acting as an alternative concentrating mechanism towards on-chip ultratrace detection. This preconcentration system requires no external energy source, hence facilitating integration and miniaturization. Two types of microchips, containing chevron and heart-shaped geometries, were used to demonstrate the trapping and confinement of micromotors due to the steric hindrance that these physical boundaries cause on their movement.

\section{Conclusions}

The article has reviewed recent advances in using self-propelled nanomotors for bioaffinity sensing and for isolating biological targets from complex samples. The new motor-based sensing approach relies on new capabilities of modern nano/microscale motors. Such sensing strategy is based on the motility of artificial nanomotors, functionalized with different bioreceptors, for advanced biosensing applications. The new binding and transport capabilities of receptormodified microtransporters have led to new routes for isolating and detecting biologically important targets. Particular attention has been given to novel sensing protocols based on selfpropelled tubular engine microtransporters for 'on-the-fly' binding and isolation of biological targets (e.g., proteins, nucleic acids, bacteria and cancer cell targets) from complex samples.

Movement of the bioreceptor around the sample leads to a fundamentally new approach for bioaffinity assays and bioseparations that greatly enhances the binding efficiency and sensitivity. The new approach addresses the low binding efficiency associated with the slow analyte transport common in existing bioaffinity sensors based on quiescent sample droplets. The continuous motility of receptor-functionalied nanomotors increases the likelihood of targetreceptor contacts, and hence greatly enhances the sensitivity of such assays. The unique features 
of the new motion-based bio-isolation platform make it an extremely attractive alternative for current bioaffinity sensing and isolation protocols. The incorporation of the new microenginebased target biosensing and isolation protocols into microchannel networks could lead to powerful analytical microchip operations. Such new capabilities have been demonstrated over the past 5 years in connection to a variety of biomolecular interactions. Two micromotor-based routes have been particularly useful for enhancing bioaffinity sensing, including movement of the bioreceptor-functionalized motor around the sample and the mixing associated with the motion of unmodified micromotors. Both of these micromotor schemes can greatly enhance analyte-receptor interactions. Further improvements in the sensitivity are expected through coupling with signal amplification strategies.

Progress in the field will likely come from integration of recent advances into more functional and complete devices. The new biosensing and isolation capabilities of functionalized microtransporters open up new opportunities of using these tiny machines in diverse real-life biosensing applications in the fields of biomedical diagnostics, environmental monitoring, defense applications or food safety. These fields will greatly benefit from the motor ability to move autonomously and expedite the binding, isolation, removal and transport of biological targets of different scales from unprocessed biological samples. Eventually, we expect to realize self responsive motors that respond to target analytes via chemotaxis and move along analyte gradient. These tiny motors should also allow new and novel sensing applications in previously in accessible environments. As future nanomachines become more functional and advanced they are expected to perform more demanding analytical tasks. The exciting research field of nanomachines is thus expected to lead to the development of innovative biosensing strategies.

\section{Acknowledgements}

This work was supported by the Defense Threat Reduction Agency-Joint Science and Technology Office for Chemical and Biological Defense (Grant no. HDTRA1-13-1-0002). The authors would like to acknowledge the important contributions of the UCSD Nanobioelectronic 
(NBE) team members to the development of motion-based sensing and advanced micromotors. (Reviewer 1 and Reviewer 2)

\section{References}

Adey, N.B.; $\quad$ Lei, M.; Howard, M.T.; Jensen, J.D.; Mayo, D.A.; Butel, D.L.; Coffin, S.C.; Moyer, T.C.; Slade, D.E.; Spute, M.K.; Hancock, A.M.; $\quad$ Eisenhoffer, G.T.; $\quad$ Dalley, B.K., McNeely, M.R. 2002, Anal. Chem. 74, 6413-6417. (Reviewer 1, Comment 1).

Balasubramanian, S.; Kagan, D.; Manesh, K.M. ; Calvo-Marzal; P.; Flechsig, G.U.; Wang, J. 2009, Small, 5, 1569-1574. (Reviewer 1, Comment 2).

Balasubramanian, S. Kagan, D., Hu, C.J. Campuzano, S., Lobo-Castañon, M.J., Lim, N., Kang, D.Y., Zimmerman, M., Zhang, L., Wang. J. 2011, Angew. Chem. Int. Ed., 50, 4161-4164.

Briand, E., Salmain, M., Herry, J.M., Perrot, H., Compère, C., Pradier, C.M., 2006, Biosens. Bioelectron., 22, 440-448.

Burdick, J., Laocharoensuk, R., Wheat, P.M. Posner, J.D., Wang, J. 2008, J. Am. Chem. Soc., 8164-8165.

Campuzano, S., Kagan, D., Orozco, J., Wang, J. 2011, Analyst, 136, 4621-4630.

Campuzano, S., Orozco J., Kagan, D., Guix, M. Gao, W., Sattayasamitsathit, S. Claussen, J.C., Merkoçi A., Wang, J., 2012, Nano Lett., 12, 396-401.

Cosnier, S. 2005, Electroanalysis, 17, 1701-1715.

Fischer, T., Agarwal, A., Hess. H., Nat. Nanotech., 2009, 4, 162-166.

Gao, W., Sattayasamitsathit, S., Orozco J., Wang, J. 2011, J. Am. Chem. Soc., 133, 1186211864.

Gao, W. Uygun, A., Wang, J. 2012, J. Am. Chem. Soc. 134, 897-900.

Garcia, M., Orozco, J., Guix, M., Gao, W., Sattayasamitsathit, S., Escarpa, A., Merkoci, M., Wang, J. 2013, Nanoscale, 5, 1325-1331.

Guix, M., Carmen, C., Mayorga-Martinez, C. C., Merkoçi, A. 2014, Chem. Rev. 114, 62856322.

Hiyama, S., Inoue, T., Shima, T., Moritani, Y., Suda, T., Sutoh, K. 2008, Small, 4, 410-415. 
Huang, C., Shen, X. 2014, Chem. Commun ., 2646-2649.

Kagan, D., Campuzano, S., Balasubramanian, S., Kuralay, F., Flechsig G.U., Wang. J. 2011, Nano Letters, 11, 2083-2087.

Kagan, K., Benchimol, M.J., Claussen, J.C., Chuluun-Erdene, E., Esener, S., Wang, J. 2012, Angew. Chem. Int. Ed. Engl. 51, 7519-7522.

Kuralay, F., Sattayasamitsathit, S., Gao, W., Uygun, A., Katzenberg, A., Wang, J., 2012, J. Am. Chem. Soc , 134, 15217-15220.

Levicky, T. M. Herne, M. J. Tarlov and S. K. Satija, 1998, J. Am. Chem. Soc. 120, 97879792Links, D.A. 2012 Lab Chip 12, 133-138. (Reviewer 1, Comment 1)

Mei, Y., Huang, G., Solovev, A.A., Bermúdez Ureña, E., Mönch, I., Ding, F., Reindl, T., Fu, R.K.Y., Chu, P.K., Schmidt, O.G., 2008, Adv. Mater., 20, 4085-4090.

Mei, Y., Solovev, A.A. Sanchez, S., Schmidt. O.G. , 2011 Chem. Soc. Rev., 40, 2109-2119.

Merkoçi, A. 2012, Electroanalysis, 25, 15-27.

Morales-Narváez, E., Guix, M., Medina-Sánchez, M., Mayorga-Martinez, C.C., Merkoçi, A. 2014, Small, 10, 2542-2548.

Nguyen, K., Minteer, S. 2015, Chem. Comm. 51, 4782-4784

Orozco, J., Campuzano, S., Kagan, D., Ming, Z., Gao, W., Wang, J., 2011, Anal Chem. 83, 7962-7969.

Orozco, J., Cortes, A., Cheng, G., Sattayasamitsathit, S., Gao, W., Feng, X., Shen, Y., Wang, J. 2013, J. Am. Chem. Soc. , 135, 5336-5339.

Orozco, J., Pan, G., Sattayasamitsathit, S., Galarnyk, M., Wang, J. 2015, Analyst, 140,14211427.

Orozco, J., Jurado-Sanchez, B., Wagner, G., Gao, W., Vazquez-Duhalt, R., Sattayasamitsathit, S., Galarnyk, M., Cortes, A., Saintillan, D., Wang, J. 2014, Langmuir, 30, 5082-5087.

Ozin, G.A.; Manners, I.; Fournier-Bidoz, S.; Arsenault, A., 2005, Adv. Mater., 17, 3011-3018.

Paxton, W.F., Kistler, K. C., Olmeda, C.C., Sen, A., St. Angelo, S.K., Cao, Y., Mallouk, T.E., Lammert, P.E., Crespi, V.H. 2004, J. Am. Chem. Soc., 126, 13424-13431.

Peyer, K.E., Zhang, L., Nelson, B.J. 2013, Nanoscale, 5, 1259-1272.

Pumera, M. 2010, Nanoscale 2, 1643-1649. (Reviewer 2). 
Rao, K.J.; Li, F.; Meng, L.; Zheng, H.; Cai, F.; Wang, W., 2015, Small, DOI: 10.1002/smll.20140362. (Reviewer 1, Comment 2).

Restrepo-Pérez, L., Soler, L., Martínez-Cisneros, C., Sánchez, S., Schmidt, O.G., 2014, Lab on a Chip 14, 2914-2917.

Sanchez, S., Solovev, A.A., Schulze, S., Schmidt, O.G., 2011(a), Chem. Commun., 47, 698-700.

Sanchez, S., Solovev, A.A., Harazim, S.M., Schmidt, O.G., 2011 (b) J. Am. Chem. Soc., 133, 701-703.

Sanchez, S., Soler, L., Katuri, J. 2015, Angew. Chem. Int. Ed. 54, 1414-1444.

Schmidt, C., Vogel, V., 2010, Lab on a Chip, 10, 2195-2198.

Sheehan, P.E.; Whitman, L.J. 2005, Nano Lett., 5, 803-807.

Simmchen, J.; Baeza, A.; Ruiz, D.; Esplandiu, M.J.; Vallet-Regí, M. 2012, Small 8, 2053-2059.

(Reviewer 1, Comment 4).

Solovev, A.A., Mei, Y., Bermúdez Ureña, E., Huang, G., Schmidt, O.G., 2009, Small, 5, 16881692.

Steel, A.B., Herne, T.M., Tarlov, M.J., 1998, Anal. Chem., 70, 4670-4677.

Vale, R.D. 2013, Cell, 112, 467-480.

van den Heuvel, M.G.L. Dekker, C. 2007, Science, 317, 333-336.

Vilela, D., Orozco, J., Cheng, C., Sattayasamitsathit, S., Galarnyk, M., Kan, C., Wang, J., Escarpa, A. 2014, Lab on Chip, 14, 3505-3509.

Wang, J., 2006, Biosensors Bioelectronics, 21, 1887-1892.

Wang, J.; Manesh, K.M. 2010 Small, 6, 338-345. (Reviewer 1, Comment 2).

Wang, J. Nanomachines: Fundamentals and Applications, Wiley-VCH, Weinheim, Germany, 2013, ISBN 978-3-527-33120-8.

\section{Figure Captions}


Figure 1. Micromotor-based routes for enhancing bioaffinity sensing. (A) Movement of the bioreceptor-functionalized motor around a complex sample, and (B) the mixing and accelerated analyte-receptor interactions induced by the motion of unmodified micromotors.

Figure 2. Motor-based isolation of different types of target biomolecules in connection to diverse surface receptors and biomolecular interactions. Top: Schematic of tubular microengines functionalized with DNA, aptamer and antibody receptors using a mixed self-assembled monolayer, along (bottom) with images of their movement with the corresponding captured targets. (Reproduced with permission from Campuzano et al, 2012).

Figure 3. Template-imprinted tubular microengines: specificity of the 'Capture-Transport' process: (a) Time-lapse images taken after 14 min navigation of the MIP-based micromotor in the $0.25 \mathrm{mg} / \mathrm{ml}$ av-FITC target protein solution. (b-d) Control experiments involving a $20 \mathrm{~min}$ navigation: Non-MIP micromotors (with PSS (b) and with SDS (c) as counter ions, respectively) in a $0.5 \mathrm{mg} / \mathrm{ml}$ av-FITC target protein solution; (d) MIP-based micromotor (based on PSS counterion) moving in a $0.5 \mathrm{mg} / \mathrm{ml}$ AIgG-FITC non-target protein solution. Scale bar, $20 \mu \mathrm{m}$. (Reproduced with permission from Orozco et al, 2013).

Figure 4. Design of tubular polymer/metal microengine and its functionalization with antibody using carboxy surface functionalities and EDC/NHS coupling. (Reproduced with permission from Garcia et al, 2013).

Figure 5. Microchip immunoassay protocol based on the movement of antibody-functionalized synthetic microtransporters and a microsphere tag. (a-c) Anti-IgG-modified microengines approaching, contacting and capturing the IgG-antibody-modified microsphere complex, and (d) departing from the sample reservoir. (Reproduced with permission from Garcia et al, 2013).

Figure 6. A) Schematic of the functionalized microengines capturing and transporting B. globigii spores for their further destruction (B). Time-lapse images illustrating the magnetically-guided propulsion of anti-B. globigii antibody-PPy-COOH-PEDOT/Ni/Pt micromotors in a spore-containing aqueous solution (a) and functionalized micromotors transporting two target (b) and multiple target (c) spores. (Reproduced with permission from Orozco et al, 2015). 
A

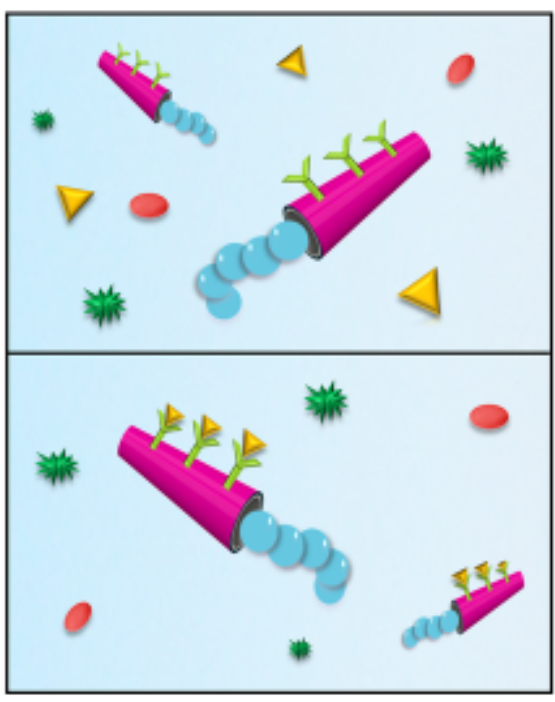

B

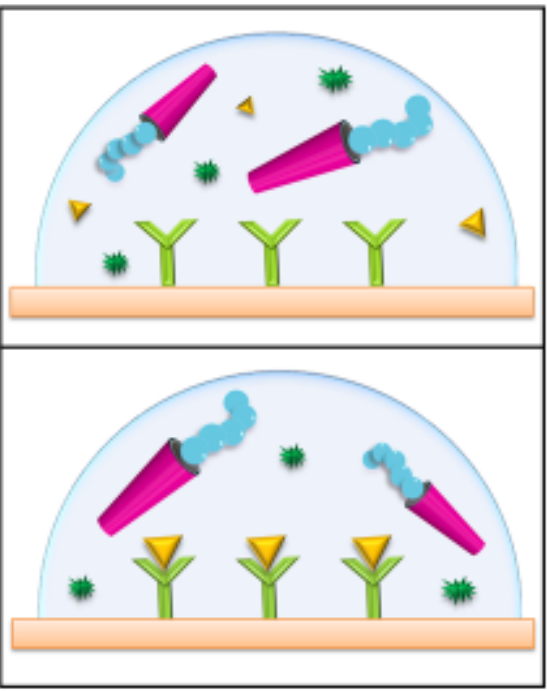

Figure 1. Micromotor-based routes for enhancing bioaffinity sensing. (A) Movement of the bioreceptor-functionalized motor around a complex sample, and (B) the mixing and accelerated analyte-receptor interactions induced by the motion of unmodified micromotors. 


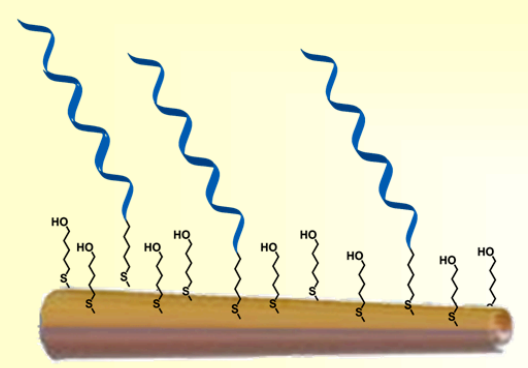

\section{DNA-modified microtransporters}

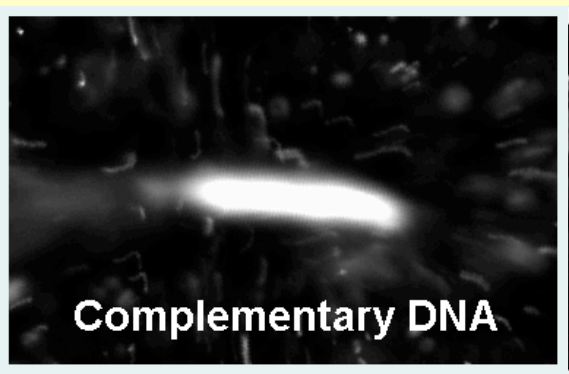

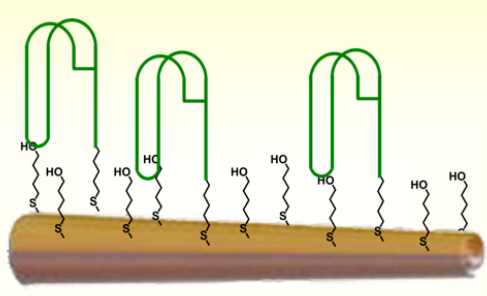

Aptamer-modified microtransporters

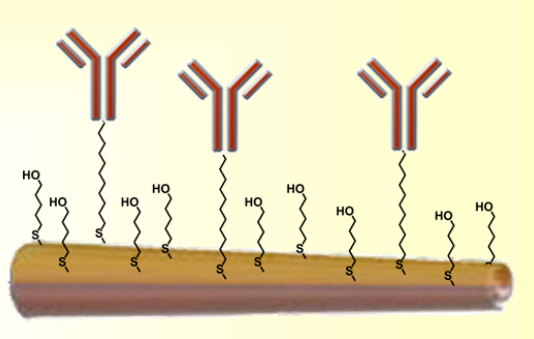

Ab-modified microtransporters
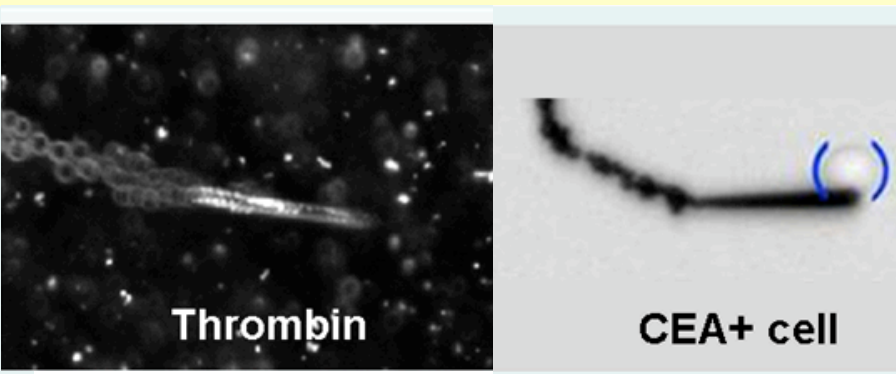

Figure 2. Motor-based isolation of different biological targets in connection to diverse surface receptors and biomolecular interactions. Top: Schematic of tubular microengines functionalized with DNA, aptamer and antibody receptors using a mixed self-assembled monolayer, along (bottom) with images of their movement with the corresponding captured targets. (Reproduced with permission from Campuzano et al, 2012). 

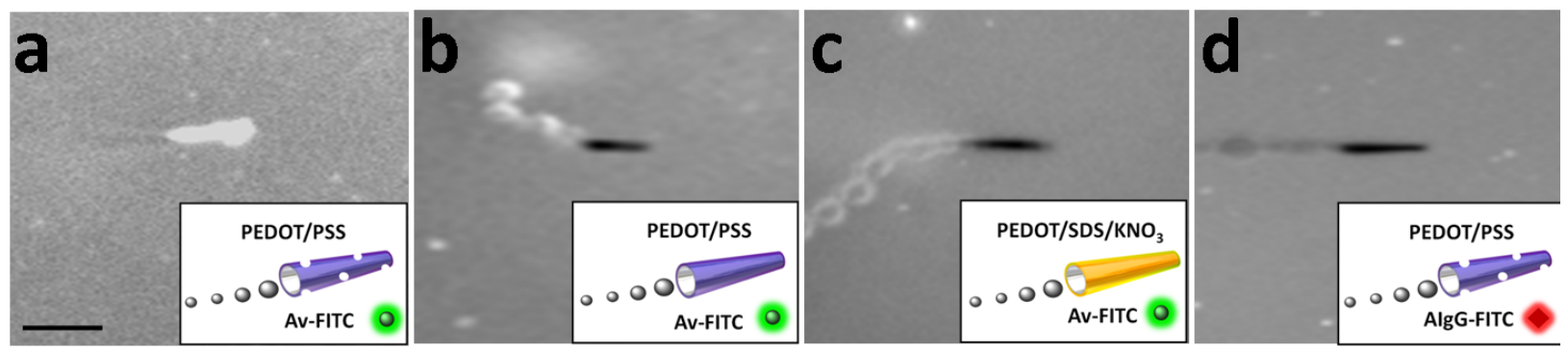

Figure 3. Template-imprinted tubular microengines: specificity of the 'Capture-Transport' process: (a) Time-lapse images taken after 14 min navigation of the MIP-based micromotor in the $0.25 \mathrm{mg} / \mathrm{ml}$ av-FITC target protein solution. (b-d) Control experiments involving a $20 \mathrm{~min}$ navigation: Non-MIP micromotors (with PSS (b) and with SDS (c) as counter ions, respectively) in a $0.5 \mathrm{mg} / \mathrm{ml}$ av-FITC target protein solution; (d) MIP-based micromotor (based on PSS counterion) moving in a $0.5 \mathrm{mg} / \mathrm{ml} \mathrm{AIgG-FITC} \mathrm{non-target} \mathrm{protein} \mathrm{solution.} \mathrm{Scale} \mathrm{bar,} 20 \mu \mathrm{m}$. (Reproduced with permission from Orozco et al, 2013).

\section{Microengine surface modification}

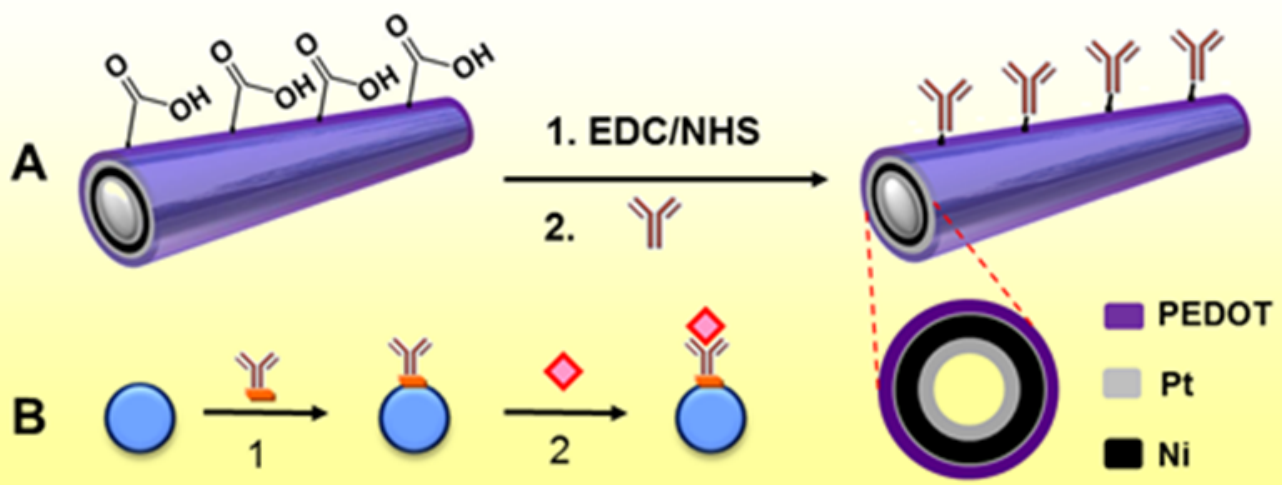

\section{PEDOT/COOH-PEDOT microengine}

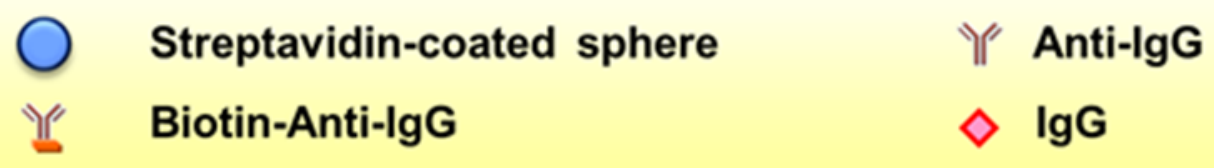

Figure 4. Design of tubular polymer/metal microengine and its functionalization with antibody using carboxy surface functionalities and EDC/NHS coupling. (Reproduced with permission from Garcia et al, 2013). 

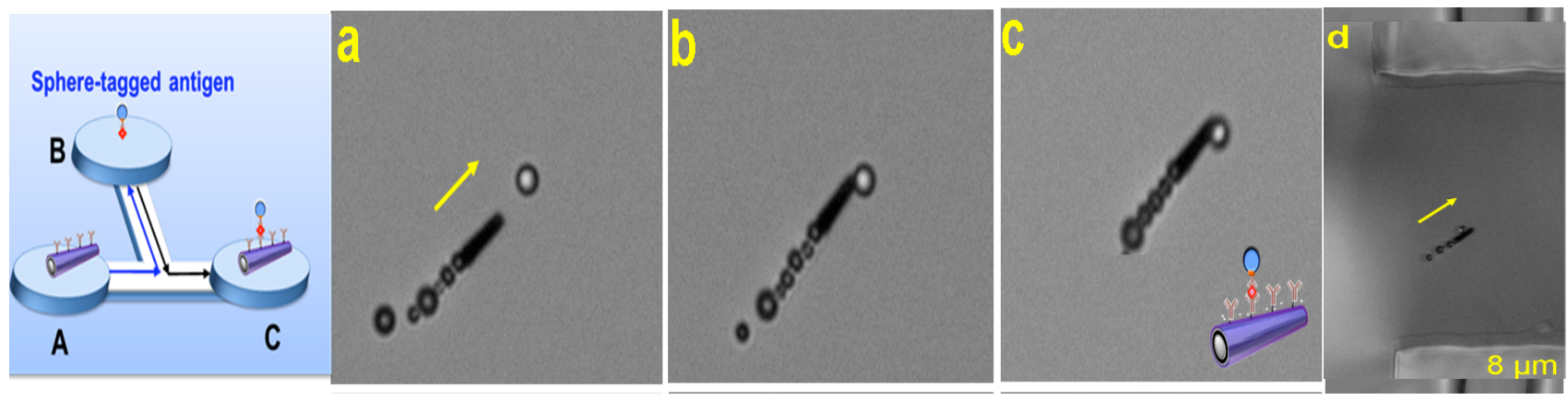

Figure 5. Microchip immunoassay protocol based on the movement of antibody-functionalized synthetic microtransporters and a microsphere tag for visualizing the binding event. (a-c) AntiIgG-modified microengines approaching, contacting and capturing the IgG-antibody-modified microsphere complex, and (d) departing from the sample reservoir. (Reproduced with permission from Garcia et al, 2013).

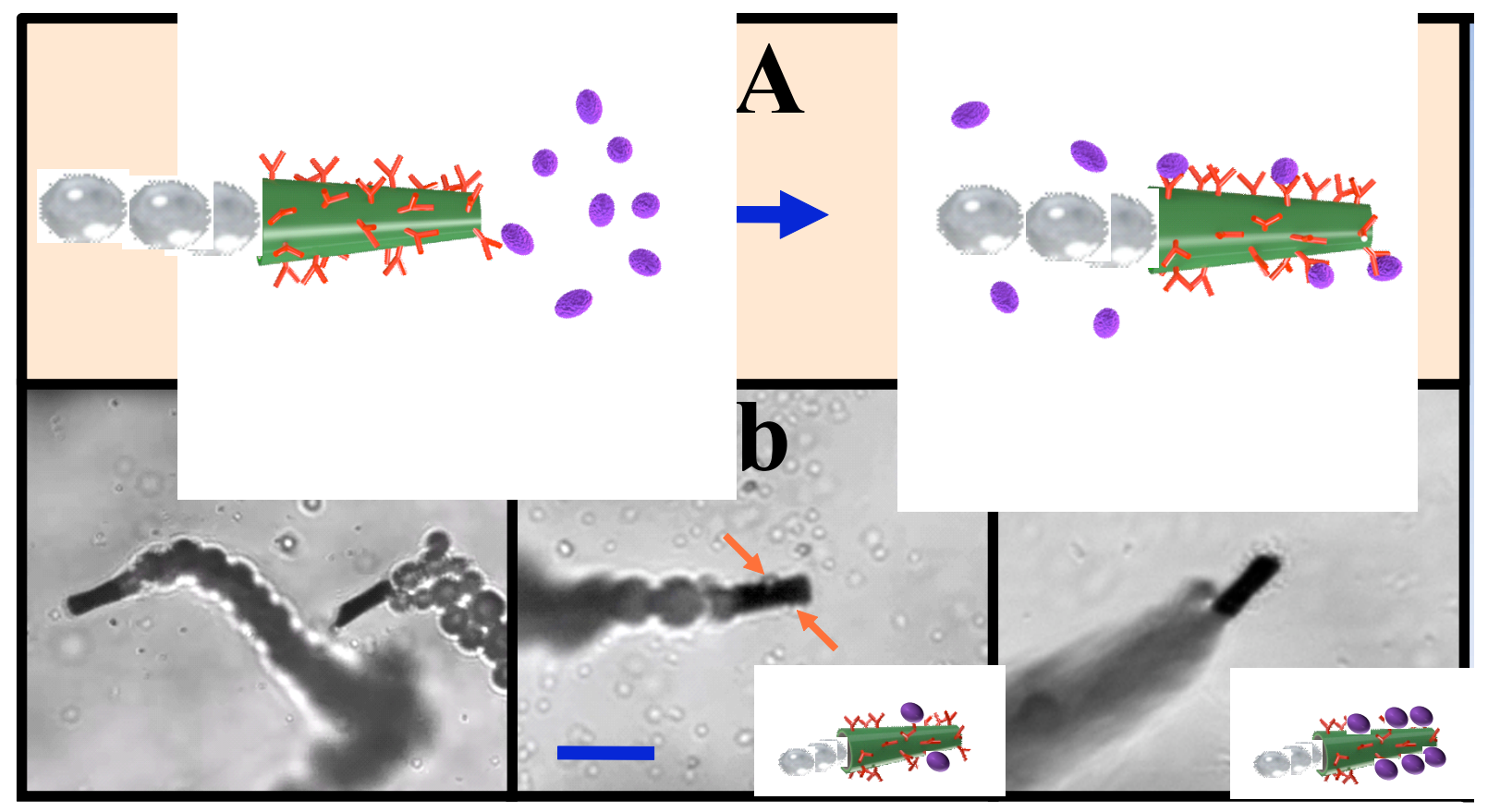

Figure 6. A) Schematic of the functionalized microengines capturing and transporting B. globigii spores for their further destruction (B). Time-lapse images illustrating the magnetically-guided propulsion of anti-B. globigii antibody-PPy-COOH-PEDOT/Ni/Pt micromotors in a spore-containing aqueous solution (a) and functionalized micromotors transporting two target (b) and multiple target (c) spores. (Reproduced with permission from Orozco et al, 2015). 


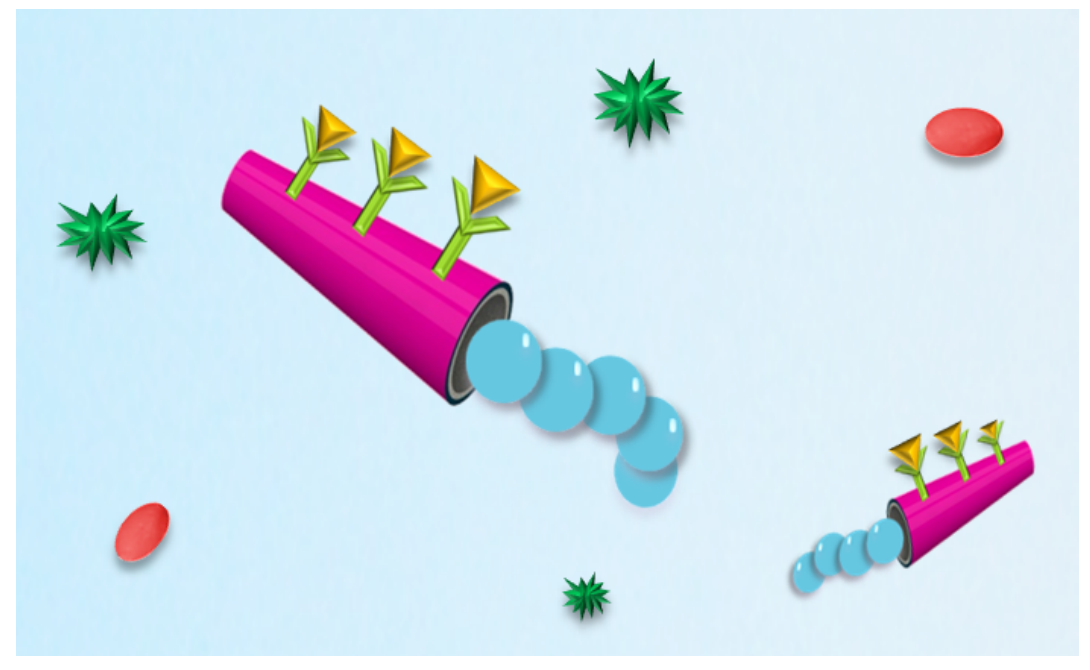

TOC. Bioaffinity sensing based on receptor-functionalized microengines. 


\section{Biographical sketch}

Joseph Wang is Distinguished Professor, SAIC Endowed Chair and

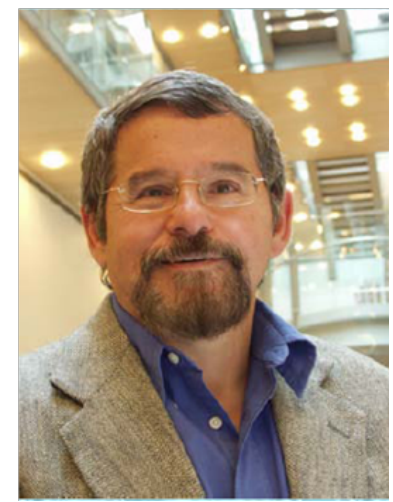

Chair in the Department of Nanoengineering at the University of California, San Diego (UCSD). He received his Ph.D. from the Technion in 1978 and five additional honorary doctor titles from Spain, Argentina and China. He held a Regents Professorship and a Manasse Chair positions at NMSU, and served as the Director of Center for Bioelectronics and Biosensors of Arizona State University (ASU). Prof. Wang has published 950 papers and he holds 20 patents. He is the Editor-in-Chief of Electroanalysis. His scientific interests are concentrated in the areas of nanobiotechnology, biosensors, bioelectronics, and nanomachines. 